CS 2018.01.01.6

Bionatura Conference Series Vol 1. No 1.2018

Publicación del IV CONGRESO INTERNACIONAL DE BIOTECNOLOGÍA Y BIODIVERSIDAD IV CIBB Y XV FORO INTERNACIONAL BANANERO

INVESTIGATION / RESEARCH

Previos / Index / Next

\title{
Extracción de Carotenos de Cáscara de Mango (Mangifera indica) con mezclas de Solventes y Extracción Asistida
}

Extraction of Carotenes from Mango Peel (Mangifera indica) with mixtures of Solvents and Assisted Extraction

Calderón Sara ${ }^{1}$; Vera Edwin ${ }^{1}$

Disponible en: http://dx.doi.org/10.21931/RB/CS/2018.01.01.6

\begin{abstract}
The extraction of carotenoids with lyophilized peel of three varieties of mango was carried out as matrix: Tommy Atkins, Haden and Kent. The following physical and chemical characteristics were evaluated: color, humidity, soluble solids, $\mathrm{pH}$, and water retention capacity (CRA). The Tommy Atkins variety presented the highest values of moisture, $\mathrm{pH}$, soluble solids, and (CRA), while the Haden variety stands out in the content of ashes and total carotenes. It was used for the extraction of carotenes mixtures of hexane: heptane: ethanol in different concentrations where heptane was the solvent with the highest extraction performance of carotenes, with $27.24 \%$ with regard to the method assisted by ultrasound. For the enzymatic treatment, we worked at a temperature of $35^{\circ} \mathrm{C}$, with two enzymatic cocktails with constant agitation for 30 and $60 \mathrm{~min}$. The GRANOZYME PTE 100 enzyme complex had an extraction yield equal to $60.12 \%$. The ultrasound-assisted extraction (UAE) was performed in an ultrasonic processor for 10, 15 and $30 \mathrm{~min}$ at a power of $750 \mathrm{~W}$, frequency of $20 \mathrm{kHz}$ and a duty cycle of $60 \%$ at room
\end{abstract}


temperature. After $30 \mathrm{~min}$, the highest extraction yields were obtained. The Haden variety is an interesting source of carotenes.

Key words: Mango, Carotenes, Ultrasound-assisted extraction, Enzymatic hydrolysis.

\section{RESUMEN}

Se realizó la extracción de carotenos con cáscara liofilizada de tres variedades de mango como matriz: Tommy Atkins, Haden y Kent. Se evaluaron las siguientes características físicas y químicas: color, humedad, sólidos solubles, $\mathrm{pH}$, sólidos solubles y capacidad de retención de agua (CRA). La variedad Tommy Atkins presentó los valores más altos de humedad, $\mathrm{pH}$, sólidos solubles, y (CRA), mientras que la variedad Haden destaca en el contenido de cenizas y carotenos totales. Se utilizó para la extracción de carotenos mezclas de hexano: heptano: etanol en diferentes concentraciones donde el heptano fue el solvente con mayor rendimiento de extracción de carotenos, con un 27,24\% respecto al método asistido por ultrasonido. Para el tratamiento enzimático, se trabajó a una temperatura de $35^{\circ} \mathrm{C}$, con dos cocteles enzimáticos con agitación constante durante 30 y 60 min. El complejo enzimático GRANOZYME PTE 100 tuvo un rendimiento de extracción igual a 60,12 \%. La extracción asistida por ultrasonido (UAE) se realizó en un procesador ultrasónico durante 10, 15 y 30 min a una potencia de $750 \mathrm{~W}$, frecuencia de $20 \mathrm{kHz}$ y un ciclo de trabajo del $60 \%$ a temperatura ambiente. A los $30 \mathrm{~min}$ se obtuvo los mayores rendimientos de extracción. La variedad Haden, es una fuente interesante de carotenos.

Palabras claves: Mango, Carotenos, Extracción asistida por ultrasonido, Hidrólisis enzimática.

\section{INTRODUCCIÓN}

El mango se ha convertido en una fruta tropical no tradicional importante en el Ecuador. La mayor producción de mango se encuentra en la provincia del Guayas, las principales variedades cultivadas en el Ecuador son Tommy Atkins, Kent, Ataulfo y Haden. Sin embargo, su producción genera desechos desde su cultivo en 
finca hasta su procesamiento. El margen estimado de rechazo y desperdicio en la producción de mango en el Ecuador es del $15 \%{ }^{1}$

Durante el procesamiento del mango la cáscara es el principal subproducto; no obstante como no es aprovechada en la industria alimentaria, se descarta como un residuo y se convierte en una fuente de contaminación ${ }^{2}$. Sin embargo, los desechos generados en la cadena de producción del mango podrían ser aprovechados para la obtención de compuestos bioactivos.

Según la variedad, la cáscara de mango representa del 15 al $18 \%$ del peso total del fruto y es una fuente rica de compuestos bioactivos, entre ellos los carotenos que presentan actividad antioxidante ${ }^{3}$. Los compuestos antioxidantes sintéticos utilizados en la industria de alimentos tienen algunos efectos secundarios y algunos son cancerígenos; por tal razón el interés en antioxidantes de origen natural ha aumentado por su valor nutricional y potencial terapéutico ${ }^{2}$. En la naturaleza se han identificado más de 600 carotenoides y sólo aproximadamente 50 de estos compuestos se encuentran comúnmente en los alimentos y son metabolizados por el cuerpo humano.

Los carotenoides son los responsables de los colores claros (amarillo a rojo) en los frutos. Estos compuestos son generalmente insolubles en agua y solubles en disolventes orgánicos como acetona, alcohol, éter etílico, cloroformo y acetato de etilo. Los carotenos son fácilmente solubles en el éter de petróleo, hexano y tolueno. ${ }^{4-5}$

En un estudio determinó que las cáscaras de mangos maduros son 4-8 veces más ricos en carotenoides que las parcialmente maduras ${ }^{2}$. En la cáscara de mango maduro según se reporta un contenido de carotenoides entre 1520 a $3337 \mathrm{ug} / \mathrm{g}^{2}$; en otro estudio con cáscaras de mango de la india liofilizadas ${ }^{6}$, se encontraron que el nivel más alto de carotenoides totales fue de $3092 \pm 98 \mathrm{ug} / \mathrm{g}$ y que presentaban potenciales actividades antioxidantes.

Los carotenoides totales en la cáscara de mango se incrementan significativamente con la maduración del fruto ${ }^{4}$. El perfil de carotenoides mostró que los principales 
compuestos son $\beta$-caroteno y violaxantina; la variedad de mango Janardhan Pasand (rojo) presentó el 91,89 \% de $\beta$-caroteno y la variedad Amrapali (verde) el $26,51 \% \beta$-caroteno. Resultados similares se encontraron mediante análisis por HPLC, donde se reporta que los carotenoides presentes en la cáscara de mango verde y maduro de la variedad Badami son violaxantina, luteína y $\beta$-caroteno, siendo el $\beta$-caroteno el que se encuentra en mayor cantidad. ${ }^{6}$

El mango se ha identificado como una fuente de $\beta$-caroteno, sin embargo, la mayor parte de las investigaciones se han centrado en la pulpa de los frutos, pero el estudio de la extracción de estos pigmentos en la cáscara del mango es limitado. ${ }^{4}$

La extracción de carotenos extiende las alternativas para la valorización de los desechos. No obstante, la extracción con disolventes orgánicos genera residuos que ocasionan un problema ambiental, por lo que es necesario mejorar el rendimiento de extracción mediante pretratamientos ${ }^{7}$. En la extracción de compuestos bioactivos se han evaluado métodos con fluidos supercríticos (FSC), microondas, ultrasonido y enzimas. 5

La extracción asistida por ultrasonido EAU incrementa las tasas de extracción del producto con un menor tiempo de procesamiento y consumo de disolventes ${ }^{8}$. En otro trabajo ${ }^{9}$ determinaron que el método de ultrasonido incrementa 40 veces el rendimiento de extracción de carotenoides con relación a una extracción directa con disolventes.

Por otra parte, la extracción asistida con enzimas hidrolíticas conduce a una liberación de los constituyentes celulares y se puede incrementar el rendimiento de extracción ${ }^{10}$. El uso de un complejo enzimático apropiado degrada los tejidos celulares, libera el compuesto de interés en la fase líquida y mejora la calidad del producto. $^{11}$

La presente investigación propone evaluar procesos de extracción de carotenos de cáscara de mango mediante solventes de fácil recuperación y reutilización, empleando la extracción asistida por ultrasonido y cocteles enzimáticos con el fin de aumentar el rendimiento de la extracción, y valorizar la cáscara de mango como 
una fuente importante de compuestos bioactivos.

\section{MATERIAL Y MÉTODOS}

Se utilizaron 100 frutos de mangos (Mangifera indica) en estado de madurez fisiológica y de consumo de la variedad Tommy Atkins, Haden y Kent. Los mangos fueron proporcionados por la Hacienda Carbell, ubicada en la provincia del Guayas, sector Nobol cerca al recinto Vijagual.

Los frutos se lavaron con agua clorada (100 ppm de hipoclorito de sodio). Luego se retiró la cáscara con un pelador de acero inoxidable. Se pesaron $300 \mathrm{~g}$ y se guardó en bolsas de polietileno (PE) estériles y se congelaron en un cuarto frío a $-20^{\circ} \mathrm{C}$. Posteriormente, las cáscaras se liofilizaron hasta tener peso constante, a una presión de vacío de $59 \mu \mathrm{mHg}$ y se fijó la temperatura de la placa de calentamiento del liofilizador a $40^{\circ} \mathrm{C}$; en cada lote se cargaron máximo $1000 \mathrm{~g}$ de las muestras. Las cáscaras liofilizadas se pulverizaron, se pesaron $50 \mathrm{~g}$ de muestra y se almacenó en bolsas estériles laminadas (flex up multicapa - aluminio) a $5{ }^{\circ} \mathrm{C}$ hasta su uso ${ }^{12}$.

\section{Evaluación de las características físico-químicas de las cáscaras de mango de las variedades Tommy Atkins, Haden y Kent.}

- Color de la cáscara: Se determinó con un colorímetro, las coordenadas CIE$\mathrm{L}^{*} \mathrm{a} \mathrm{b}^{*}$. Las medidas se hicieron en la cáscara fresca, para lo cual se tomó como muestra 10 mangos de cada variedad y la lectura de color se realizó en las dos caras de cada mango. ${ }^{13}$

- Contenido de cenizas: Se determinó por el método gravimétrico descrito en AOAC 923.03. Reportado en gramos de ceniza en $100 \mathrm{~g}$ de muestra.

- Humedad: Se utilizó el método gravimétrico indicado en AOAC 966.02 en estufa a $105^{\circ} \mathrm{C}$ hasta peso constante. 
- Capacidad de retención de agua (CRA): Se adicionó $1 \mathrm{~g}$ de cáscara de mango en polvo en tubos de $50 \mathrm{~mL}$ y se colocó $20 \mathrm{~mL}$ de agua destilada, se agitó durante 10 min; luego se dejó en reposo durante 24 h a temperatura ambiente, se centrifugó a $3000 \mathrm{rpm}$ durante $10 \mathrm{~min}$, se retiró el sobrenadante y se pesó el sedimento.

La CRA se determinó como (g) de agua retenido por (g) de cáscara de mango en polvo $^{12}$ de acuerdo a la ecuación (1).

$\operatorname{CRA}\left(\frac{g_{\text {agua }}}{g_{\text {cáscara }}}\right)=\frac{\text { Peso sedimento-Peso muestra seca }}{\text { Peso muestra seca }}$

- Sólidos solubles: Se determinaron con un refractómetro de mesa, escala de 0 a 85

${ }^{\circ}$ Brix, con el método AOAC 932.12.

- pH: Se realizó con un pHmetro, según el método AOAC 981.12.

\section{Evaluación del efecto de los solventes en la extracción de carotenos}

- Preparación de la muestra

A $3 \mathrm{~g}$ de cáscara de mango en polvo de la variedad Tommy Atkins en un vaso de precipitación cubierto con papel de aluminio, se adicionó bicarbonato de sodio (como agente neutralizante de posibles ácidos liberados de la muestra durante la extracción) en una relación de $1 \%(\mathrm{p} / \mathrm{p})$ con respecto al peso de la muestra y se homogenizó por 3 min.

- Extracción

Como disolventes de la extracción se utilizó mezclas de hexano: heptano: etanol en proporciones de 100:0:0, 90:0:10, 45:45:10, 0:90:10 y 0:100:0. Se adicionó el 
disolvente a trabajar con una relación sólido: solvente de 1:10 (p/v) de la siguiente manera: la mitad del disolvente se colocó en el vaso de precipitación que contiene la muestra y el álcali, se agitó por $10 \mathrm{~min}$, se dejó en reposo por 5 min y se colocó el sobrenadante (extracto) en un embudo de separación evitando el paso de sólidos. Al vaso de precipitación que contiene los sólidos se le adicionó la otra mitad del disolvente y se repitió el proceso antes descrito.

\section{- Partición y lavado}

En el embudo de separación que contiene el extracto se le adicionó agua en una relación de disolvente: agua de 1:1 (v/v), a temperatura ambiente, la adición del agua destilada fue de la siguiente manera: un tercio del agua se añadió suavemente en el embudo de separación sin agitación para evitar la formación de una emulsión, se esperó hasta visualizar las dos fases, la fase acuosa inferior se descartó. El extracto se lavó 2 veces más hasta eliminar el álcali y cualquier compuesto soluble en agua presente en el extracto ${ }^{5}$. Se recogió el extracto y se aforó a $50 \mathrm{~mL}$ en un matraz cubierto con papel de aluminio.

\section{- Cuantificación}

Se cuantificaron los carotenos totales del extracto obtenido por espectrofotometría a una absorbancia de $450 \mathrm{~nm}^{5,13}$.El contenido de carotenos totales (equivalentes a $\beta$-caroteno), expresado en $\mathrm{ug} / \mathrm{g}$ de muestra, se calculó con la Ley de Beer de acuerdo a la ecuación (2).

Carotenos Totales equivalentes a $\beta$-caroteno $\left(\frac{\mathrm{ug}}{\mathrm{g}}\right)=\frac{\mathrm{A}_{450} \mathrm{PM}^{*} \mathrm{~V} \mathrm{~F}^{*} \mathrm{f}}{\mathrm{E}^{*} \mathrm{~L} * \mathrm{~W}} * 1000$

Donde:

$\mathrm{A}_{450}=$ Absorbancia, $\mathrm{PM}=$ Peso molecular del $\beta$-caroteno $(536,89 \mathrm{~g} / \mathrm{mol}), \mathrm{V}=$ Volumen del extracto $(\mathrm{mL}), \mathrm{f}=$ Factor de dilución, $\mathrm{E}=$ Coeficiente de extinción 
molar $(1,34 \mathrm{E}+05 \mathrm{M} \quad \mathrm{cm} \quad), \mathrm{L}=$ Longitud de la celda $(\mathrm{cm})$ y $\mathrm{W}=$ Peso de la muestra $(\mathrm{g})$

La mezcla de solventes con la cual se obtuvo una mayor extracción se utilizó para analizar la extracción asistida por ultrasonido y la extracción enzimática.

\section{Evaluación del efecto del tiempo en la extracción asistida por ultrasonido (UAE).}

La muestra fue preparada como se indicó en la sección 2.2.1. Luego se adicionó el disolvente a trabajar en una relación sólido: solvente de 1:10 (p/v) y se trató con el ultrasonido durante 10,15 y $30 \mathrm{~min}$ a una potencia de $750 \mathrm{~W}$, frecuencia de 20 $\mathrm{kHz}$ y un ciclo de trabajo del $60 \%$ a temperatura ambiente.

Se preparó también una muestra control a la cual se adicionó el disolvente en la misma relación sólido: solvente $(1: 10 \mathrm{p} / \mathrm{v})$ y se agitó durante 10,15 y $30 \mathrm{~min}$ a temperatura ambiente, sin uso del ultrasonido. La solución obtenida luego de cada tratamiento se filtró y el sobrenadante obtenido (extracto) se colocó en un embudo de separación. La partición y lavado se realizaron igual que el procedimiento descrito en la sección 2.2.3.

En la cuantificación de los carotenos totales del extracto obtenido y el cálculo del rendimiento de extracción se utilizó el mismo procedimiento descrito en la sección 2.2.4.

\section{Determinación de la influencia de la hidrólisis enzimática en la extracción de carotenos.}

La muestra fue preparada como se indicó en la sección 2.2.1 y se adicionó agua destilada en una relación cáscara: agua igual a 1:10 (p/v). Para el tratamiento enzimático se utilizó baño maría a una temperatura de $35^{\circ} \mathrm{C}$, se trabajó con dos cocteles enzimáticos GRANOZYME PTE 100 y ROHAPECT® UF (dosis 50 y $100 \mathrm{ppm}$ ) con agitación constante durante 30 y $60 \mathrm{~min}$. Luego se centrifugó por 10 min a $4000 \mathrm{rpm}$ y se eliminó el sobrenadante ${ }^{14}$. Se preparó también una muestra 
control a las mismas condiciones sin enzima, para comparar la eficiencia de la hidrólisis enzimática.

Los sólidos obtenidos en la centrifugación se liofilizaron hasta tener peso constante. Se pulverizaron las muestras y se procedió con la extracción, para lo cual se siguió el mismo procedimiento descrito en la sección 2.2. Con estos resultados se realizaron igualmente los cálculos del rendimiento de extracción según se indica en la sección 2.2.

Comparación de la extracción de carotenos de las variedades Tommy Atkins, Kent y Haden.

El mejor tratamiento de extracción obtenido bajo los criterios de mejor solvente y mejor tratamiento, ultrasonido o hidrólisis enzimática se utilizó para realizar la extracción de carotenos de las cáscaras de las tres variedades de mango: Tommy Atkins, Haden y Kent.

\section{Análisis estadístico}

Los ensayos se realizaron por triplicado, a excepción de la determinación del color que se realizó 10 veces. Los resultados obtenidos se reportaron como la media \pm desviación estándar. Los análisis estadísticos se realizaron con el programa STATGRAPHICS Centurión y se analizaron mediante análisis de varianza (ANOVA) con un nivel de confianza del $95 \%$ y la diferencia significativa se definió en $\mathrm{p}<0,05$. Los intervalos se determinaron con el procedimiento de la diferencia mínima significativa (LSD) de Fisher.

\section{RESULTADOS Y DISCUSIÓN}

Evaluación de las características físico-químicas de las cáscaras de mango de las variedades Tommy Atkins, Haden y Kent.

Los resultados de las propiedades físico-químicas y de las medidas de color de 
cada una de las caras de las frutas de mango en madurez fisiológica se presentan en la Tabla 1.

\begin{tabular}{|c|c|c|c|c|}
\hline & & Tommy Atkins & Haden & Kent \\
\hline$\overline{\mathbf{L}}$ & $\begin{array}{l}\text { Cara1 } \\
\text { Cara2 }\end{array}$ & $\begin{array}{l}49,32 \pm 8,83^{\mathrm{a}} \\
53,58 \pm 9,61^{\mathrm{ab}}\end{array}$ & $\begin{array}{l}49,65 \pm 2,77^{\mathrm{a}} \\
57,04 \pm 6,88^{\mathrm{b}}\end{array}$ & $\begin{array}{l}51,89 \pm 6,08^{\mathrm{ab}} \\
53,02 \pm 5,76^{\mathrm{ab}}\end{array}$ \\
\hline $\mathbf{a}$ & $\begin{array}{l}\text { Cara1 } \\
\text { Cara2 }\end{array}$ & $\begin{array}{l}13,78 \pm 5,35^{b} \\
11,03 \pm 9,32^{b}\end{array}$ & $\begin{array}{c}25,40 \pm 4,20^{c} \\
13,62 \pm 12,46^{b}\end{array}$ & $\begin{array}{l}-9,44 \pm 3,89^{\mathrm{a}} \\
-9,74 \pm 5,41^{\mathrm{a}}\end{array}$ \\
\hline $\mathbf{b}$ & $\begin{array}{l}\text { Cara1 } \\
\text { Cara2 }\end{array}$ & $\begin{array}{l}27,48 \pm 14,22^{\mathrm{ab}} \\
33,58 \pm 12,89^{\mathrm{ab}}\end{array}$ & $\begin{array}{l}26,33 \pm 3,95^{\mathrm{a}} \\
35,64 \pm 9,24^{\mathrm{b}}\end{array}$ & $\begin{array}{l}28,11 \pm 7,56^{\mathrm{ab}} \\
29,75 \pm 6,46^{\mathrm{ab}}\end{array}$ \\
\hline \multicolumn{2}{|c|}{ "Cenizas (g/100g) } & $0,93 \pm 0,03^{b}$ & $1,01 \pm 0,05^{c}$ & $0,74 \pm 0,03^{\mathrm{a}}$ \\
\hline \multicolumn{2}{|c|}{$\begin{array}{c}\text { *Humedad } \\
(\%)\end{array}$} & $79,17 \pm 0,58^{c}$ & $72,16 \pm 0,43^{a}$ & $75,65 \pm 0,27^{b}$ \\
\hline \multicolumn{2}{|c|}{$\mathrm{pH}$} & $4,94 \pm 0,01^{b}$ & $4,89 \pm 0,06^{b}$ & $4,72 \pm 0,03^{a}$ \\
\hline \multicolumn{2}{|c|}{$\begin{array}{l}\text { Sólidos solubles } \\
\text { ( }{ }^{\circ} \text { Brix) }\end{array}$} & $6,73 \pm 0,06^{c}$ & $6,53 \pm 0,12^{b}$ & $5,47 \pm 0,12^{a}$ \\
\hline \multicolumn{2}{|c|}{$\begin{array}{c}\text { CRAC (g agua/g } \\
\text { cáscara seca) }\end{array}$} & $4,87 \pm 0,13^{b}$ & $4,12 \pm 0,45^{a}$ & $3,94 \pm 0,16^{\mathrm{a}}$ \\
\hline
\end{tabular}

*Análisis realizado en cáscara fresca de cada variedad

${ }^{* *}$ Carotenos totales equivalentes a $\beta$-caroteno

Valores corresponden a: media \pm desviación estándar, (n=9, $n=60$ par L, $a, b)$,

Letras minúsculas diferentes indican diferencias significativas en la variedad de cada mango $(p<0,05)$.

L (luminosidad): 0 (negro) a 100 (blanco); a (verde a rojo): + a (hacia el rojo), - a (hacia el verde); $b$ (azul a amarillo): +b (hacia el amarillo), -b (hacia el azul)

\section{Tabla 1. Características físico-químicas de las cáscaras de mango de las variedades Tommy Atkins, Haden y Kent}

En cuanto al color, los resultados indican que las dos caras de las variedades Tommy Atkins y Kent no presentan diferencias significativas, mientras que para la variedad Haden existen diferencias significativas para los tres parámetros $L^{*} a^{*} b^{*}$. 
Estos resultados sugieren que las variedades Tommy Atkins y Kent tienen un color uniforme, mientras que la variedad Haden tiene diferentes tonalidades.

El valor $L$, que representa el brillo, fue significativamente mayor para la variedad Haden, seguido por la variedad Tommy Atkins y el menor valor se registró en la variedad Kent.

La variedad Haden registró un valor positivo de $a$ que indica enrojecimiento, al igual que la variedad Tommy Atkins, mientras que la variedad Kent mostró valores negativos de $a$ que indica verdor. El valor $b$ fue positivo en las tres variedades lo cual indica amarillez, el valor más alto fue en la variedad Haden, y no existió diferencia significativa entre las variedades Tommy Atkins y Kent.

Otros autores ${ }^{4}$ reportaron observaciones similares para tres variedades de mango de coloración roja, amarilla y verde (Janardhan Pasand, Arka Anmol y Amrapali), que presentaron valores de: $L$ entre 45,76 a 62,86 ; $a$ entre $-8,66$ a 29,92 y $b$ entre de 28,6 a 48,86 .

El color de la cáscara de estas variedades de mango es similar a las tres variedades analizadas Tommy Atkins, Haden y Kent, que puede atribuirse al grado de madurez de los mangos, ya que todos los mangos fueron cosechados en madurez fisiológica y madurados hasta la madurez de consumo a condiciones ambientales.

La variedad Tommy Atkins presentó los valores más altos de humedad en cáscara fresca y valores altos de $\mathrm{pH}$, sólidos solubles, y capacidad de retención de agua (CRA en la cáscara liofilizada en polvo), mientras que la variedad Haden destaca en el contenido de cenizas y carotenos totales.

Los valores de humedad son significativamente diferentes en las tres variedades y presentaron valores menores a los reportados: $83,37 \%$ en mango Tommy Atkins ${ }^{15}$. Estas variaciones podrían deberse al grado de madurez y las variedades de mango analizadas en cada estudio; en otros estudios los valores de humedad de la cáscara reportados son 79,8 \% en mango Tommy Atkins y 75,38 \% en mango hiacha ${ }^{16}$, siendo estos resultados similares a los obtenidos en este estudio. 
En el contenido de cenizas existen diferencias significativas en las tres variedades.

En estudios realizados en cáscara de mango variedad hilacha ${ }^{16}$, se reportó un contenido de cenizas de $0,67 \mathrm{~g} / 100 \mathrm{~g}$, siendo este resultado menor a los obtenidos en este trabajo, esto se debe a que la cáscara mango hilacha tiene $56,27 \%$ de fibra insoluble en base seca.

El pH de la cáscara de mango liofilizada no presentó diferencia significativa entre las variedades Tommy Atkins y Haden. De acuerdo a los valores obtenidos, las tres variedades presentan una cáscara ligeramente ácida, y se obtuvo resultados mayores que los reportados en cáscara de mango Tommy Atkins ${ }^{15}$.

En términos del contenido de sólidos solubles existe diferencia significativa entre las variedades de cáscara analizadas. La variedad Tommy Atkins presentó el mayor valor, sin embargo, los resultados obtenidos son 2 a 3 veces menor que el contenido de sólidos solubles reportados para la pulpa de mango ecuatoriano. ${ }^{17}$

Los valores obtenidos de CRA fueron menores a los valores reportados en bibliografía $^{12}$ en cáscaras de mango Tommy Atkins, pero son similares a los valores reportados en otros trabajos, donde se reporta valores de 4,98 g agua/g cáscara seca para cáscaras liofilizadas de mangos maduros de la variedad Tommy Atkins $^{15}$. Para la CRA, la variación de resultados pudo deberse al diámetro de partícula de la cáscara liofilizada ${ }^{12}$, con menores diámetros los valores de CRA aumentan.

\section{Evaluación del efecto de los solventes en la extracción de carotenos}

Al realizar la extracción de los carotenos de la cáscara de mango variedad Tommy Atkins con diferentes mezclas de los solventes hexano, heptano y etanol, se encontró que el heptano tiene una mayor recuperación de carotenos (Tabla 2), seguido por la solución heptano: etanol 90:10 y el hexano. Estos resultados se explican porque el heptano es un disolvente con una solubilidad de apenas 0,0003 $(\% \mathrm{p} / \mathrm{p})$ en agua, es más hidrófobo que el hexano y más selectivo para el $\beta$ caroteno. En cuanto al hexano la solubilidad de ciertos carotenos y sustancias liposolubles es menor por lo que se suele realizar extracciones múltiples y e usa 
una cantidad mayor de solvente. ${ }^{18}$

\begin{tabular}{|l|l|}
\hline Solventes & Carotenos totales (ug/g) \\
\hline Heptano $100 \%$ & $141,43 \pm 2,61^{\mathrm{d}}$ \\
\hline Hexano $100 \%$ & $101,72 \pm 2,51^{\mathrm{b}}$ \\
\hline $\begin{array}{l}\text { Heptano/Hexano/Etanol } \\
\text { 90/0/10 }\end{array}$ & $120,26 \pm 3,55^{\mathrm{c}}$ \\
\hline $\begin{array}{l}\text { Heptano/Hexano/Etanol } \\
\text { 45/45/10 }\end{array}$ & $91,95 \pm 3,29^{\mathrm{a}}$ \\
\hline $\begin{array}{l}\text { Heptano/Hexano/Etanol } \\
\text { 0/90/10 }\end{array}$ & $94,13 \pm 2,95^{\mathrm{a}}$ \\
\hline
\end{tabular}

Valores corresponden a: media \pm desviación estándar, $(n=15)$,

Letras minúsculas diferentes indican diferencias significativas entre las distintas concentraciones de solventes $(p<0,05)$.

Tabla 2. Resultados de la extracción de carotenos de la cáscara de mango de la variedad Tommy Atkins con diferentes solventes.

La solución de heptano/hexano/etanol (45/45/10) presentó la menor recuperación de carotenos, y no existe una diferencia significativa con la solución de heptano/hexano/etanol (0/90/10). Se observó que al añadir etanol al heptano y hexano se tiene una reducción en la recuperación de carotenos del 1,46 \% y 4,08 \% respectivamente. Esto puede explicarse debido a la baja polaridad de los carotenos que genera baja disolución en etanol. El etanol tiene mayor afinidad con los carotenoides oxigenados.

Resultados similares se obtuvo en la extracción asistida por ultrasonido de $\beta$ caroteno de Spirulina platensis ${ }^{18}$, donde se utilizaron como disolventes el hexano, el heptano y el éter dietílico, y se obtuvo el máximo rendimiento de extracción con el heptano. 


\section{Evaluación del efecto del tiempo en la extracción de carotenos asistida por}

ultrasonido (UAE).

Las pruebas de extracción asistida con ultrasonido de carotenos de la cáscara de mango Tommy Atkins, se realizaron únicamente con el heptano que fue el solvente con el mejor rendimiento de extracción (Tabla 3).

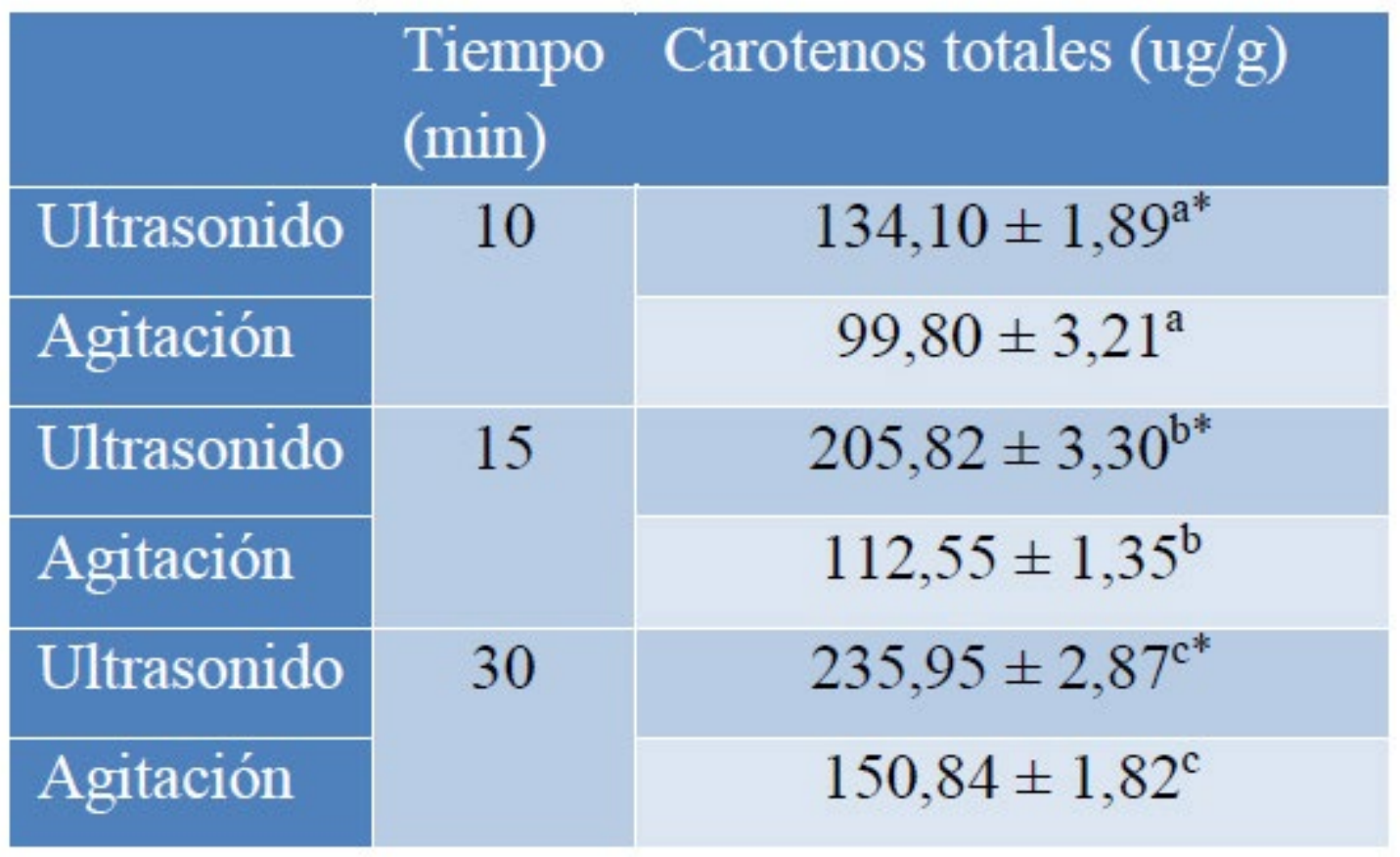

* Tratamiento con UAE

Valores corresponden a: media \pm desviación estándar, $(n=18)$,

Letras minúsculas diferentes indican diferencias significativas en el tiempo de exposición $(p<0,05)$.

Tabla 3. Resultados de la extracción de carotenos de la cáscara de mango de la variedad Tommy Atkins obtenidos en la extracción asistida por ultrasonido (UAE).

Al variar el tiempo de exposición en el ultrasonido entre 10 y 30 min, se encontró que a los 30 min se tiene el mayor rendimiento de extracción de carotenos. 
agitación y sin ultrasonido, se observa que existe una diferencia significativa. Así, por ejemplo, a los $10 \mathrm{~min}$ se tiene un aumento del $25,83 \%$ en el rendimiento de extracción cuando se usa el ultrasonido, diferencia que se incrementa con el tiempo. Esto puede deberse a que con el ultrasonido se rompe las paredes celulares por la formación de microcavidades ${ }^{8}$, y se liberan los carotenos presentes en la matriz.

\section{Determinación de la influencia de la hidrólisis enzimática en la extracción de carotenos.}

En la Tabla 4 se presentan los resultados de la extracción de carotenos con un pretratamiento enzimático. Al comparar la muestra control (sin enzimas) con los tratamientos con hidrólisis enzimática, se observa que el rendimiento de la extracción se incrementa. Así, por ejemplo, a los $30 \mathrm{~min}$ el rendimiento de la extracción se incrementó en más del 50\% con el uso de enzimas a 50 ppm.

\begin{tabular}{|c|c|c|c|}
\hline Enzima & $\begin{array}{l}\text { Tiempo }{ }^{*} \\
\text { (min) }\end{array}$ & $\begin{array}{r}\mathrm{Ce} \\
(\mathrm{ppm})\end{array}$ & Carotenos totales (ug/g) \\
\hline \multirow{2}{*}{$\begin{array}{c}\text { CONTROL } \\
\text { (Sin } \\
\text { enzima) }\end{array}$} & 30 & 0 & $85,71 \pm 0,59^{b}$ \\
\hline & 60 & 0 & $73,39 \pm 3,19^{a}$ \\
\hline \multirow{4}{*}{$\begin{array}{c}\text { GRANOZY } \\
\text { ME PTE } \\
100\end{array}$} & \multirow[t]{2}{*}{30} & 50 & $141,77 \pm 1,83^{\mathrm{f}}$ \\
\hline & & 100 & $208,06 \pm 1,86^{i}$ \\
\hline & \multirow[t]{2}{*}{60} & 50 & $120,23 \pm 1,33^{c}$ \\
\hline & & 100 & $176,97 \pm 1,35^{\mathrm{h}}$ \\
\hline \multirow{4}{*}{$\begin{array}{c}\text { ROHAPEC } \\
\text { T® UF }\end{array}$} & \multirow[t]{2}{*}{30} & 50 & $129,55 \pm 1,66^{\mathrm{e}}$ \\
\hline & & 100 & $177,51 \pm 2,53^{\mathrm{h}}$ \\
\hline & \multirow[t]{2}{*}{60} & 50 & $132,47 \pm 2,21^{d}$ \\
\hline & & 100 & $172,49 \pm 2,00^{\mathrm{g}}$ \\
\hline
\end{tabular}

Valores corresponden a: media \pm desviación estándar, $(n=30)$,

Letras minúsculas diferentes indican diferencias significativas $(p<0,05)$. 
* Tiempo de tratamiento enzimático en fase acuosa a $35^{\circ} \mathrm{C}$ en baño maría, Ce: concentración de la enzima.

\section{Tabla 4. Resultados de la extracción de carotenos de la cáscara de mango de la variedad Tommy Atkins con hidrólisis enzimática.}

En la muestra control la extracción de carotenos fue menor respecto a la extracción de carotenos con solventes (Tabla 2), probablemente por las diferentes condiciones de operación y pudo también existir pérdidas en el proceso, ya que la muestra sólida fue rehumedecida y se liofilizó nuevamente, antes de iniciar el proceso de extracción con heptano, partición y lavado que se indica en la sección 2.2.

En cuanto a los tiempos de tratamiento, para los dos cocteles enzimáticos GRANOZYME PTE 100 y ROHAPECT ${ }^{\circledR}$ UF, se encontró que el rendimiento de extracción es mayor a los $30 \mathrm{~min}$, puede ser que existe una degradación de los carotenos por su sensibilidad al oxígeno, lo que provoca que el rendimiento disminuya a los $60 \mathrm{~min}$.

En cuanto a la concentración de la enzima, se puede notar que con una concentración de 100 ppm la cantidad de carotenos extraídos es mayor que a una concentración de $50 \mathrm{ppm}$.

En las condiciones óptimas para el tratamiento enzimático (30 min y 100 ppm), con el complejo enzimático GRANOZYME PTE 100 se tuvo un rendimiento de extracción inferior respecto al método de extracción asistida por ultrasonido, comportamiento también observado por otros autores ${ }^{5}$. Con el complejo enzimático ROHAPECT® UF, el rendimiento de extracción fue más bajo.

De acuerdo al análisis estadístico, existe diferencia significativa en la extracción de carotenos para todos los tratamientos, excepto para los ensayos con $100 \mathrm{ppm}$ de GRANOZYME PTE 100 por 60 min, y 100 ppm de ROHAPECT ${ }^{\circledR}$ UF por 30 min, que son estadísticamente iguales.

Con el coctel GRANOZYME PTE 100 se obtuvo un mayor rendimiento de 
extracción, debido a que este producto tiene enzimas pectolíticas, las mismas que mejoran la extracción de compuestos de color y aroma al hidrolizar los enlaces de pectina.

En cuanto al bajo rendimiento con ROHAPECT® UF, se podría explicar mediante el contenido de pectinasas y arabanasas que tienen acción enzimática diferente: las pectinasas degradan las cadenas de pectina soluble, las arabanasas degradan las ramificaciones de la celulosa, solubilizan la pectina y mejoran el rendimiento de la pectinasa ${ }^{19}$. Sin embargo, aunque se ha determinado que la cáscara de mango maduro contiene $90-320 \mathrm{mg} / \mathrm{g}$ de pectina en base seca, no se tiene información exacta sobre la solubilidad de esta pectina ${ }^{20}$, parámetro que influiría en la eficacia del cóctel ROHAPECT ${ }^{\circledR}$ UF, en el que la actividad de la pectinasas es seguramente inferior al del coctel GRANOZYME PTE 100.

Comparación de la extracción de carotenos de las variedades Tommy Atkins, Kent y Haden.

La extracción de los carotenos de las cáscaras de las diferentes variedades de mango se realizó con las mejores condiciones de operación encontradas, es decir a temperatura ambiente, heptano como solvente (relación sólido: solvente de 1:10 p/v), con ultrasónico por $30 \mathrm{~min}$, a una potencia de $750 \mathrm{~W}$, frecuencia de $20 \mathrm{kHz}$ y un ciclo de trabajo del $60 \%$.

\begin{tabular}{|l|l|}
\multicolumn{2}{|c|}{ Carotenos totales (ug/g) } \\
\hline Tommy Atkins & $235,95 \pm 2,87^{\mathrm{b}}$ \\
\hline Haden & $249,42 \pm 1,90^{\mathrm{c}}$ \\
\hline Kent & $165,21 \pm 1,72^{\mathrm{a}}$ \\
\hline
\end{tabular}

Valores corresponden a: media \pm desviación estándar, $(n=9)$,

Letras minúsculas diferentes indican diferencias significativas en la variedad de cada mango $(p<0,05)$. 
Según los resultados presentados en la Tabla 5, la variedad de mango tiene una influencia significativa en la cantidad de carotenos extraídos. Estos resultados pueden deberse al grado de madurez de las frutas empleadas y están asociados al color característico de cada cultivar: las variedades Haden y Tommy Atkins presentan una cáscara de color rojo vivo con matices verdes y anaranjados, mientras que la variedad Kent presenta un color verde oscuro y solo tiene un sector rojizo oscuro en la cáscara.

\section{CONCLUSIONES}

Los resultados de este estudio demostraron que la variedad del mango, los pretratamientos con enzimas y el solvente empleado en la extracción tienen un impacto significativo en el rendimiento de extracción de carotenos. Además, el color de la cáscara de mango tiene relación con el contenido de carotenos.

Con el heptano se obtuvo el mayor rendimiento de extracción de carotenos. Este solvente podría ser una mejor opción para los procesos de extracción por los mejores rendimientos obtenidos.

La extracción asistida por ultrasonido de carotenos mejora la eficiencia de extracción con respecto a los métodos convencionales. El tratamiento enzimático más efectivo para la cáscara de mango fue con enzimas pectolíticas, y particularmente con el coctel GRANOZYME PTE 100 se obtuvo un mayor rendimiento de extracción cuando se usó en una concentración de 100 ppm.

Los resultados presentados muestran que las cáscaras de mango, especialmente del cultivar Haden, son una fuente interesante de carotenos.

\section{AGRADECIMIENTOS}


Los autores agradecen al Proyecto PIMI15-05 "Evaluación de la biorefinería de subproductos de procesamiento de alimentos para la obtención de compuestos de interés industrial", a Granotec Ecuador S.A y Oenobrands por proporcionar los cocteles enzimáticos para llevar a cabo el trabajo de investigación.

\section{REFERENCIAS}

1. Fundación mango Ecuador. Áreas de Cultivo. http://www.mangoecuador.org/areas-cultivo.php. Published 2016. Accessed July 20, 2009.

2. Ajila CM, Naidu KA, Bhat SG, Rao UJSP. Bioactive compounds and antioxidant potential of mango peel extract. Food Chem. 2007;105(3):982-988. doi:10.1016/J.FOODCHEM.2007.04.052

3. Okino Delgado CH, Fleuri LF. Orange and mango by-products: Agroindustrial waste as source of bioactive compounds and botanical versus commercial description-A review. Food Rev Int. 2016;32(1):1-14. doi:10.1080/87559129.2015.1041183

4. Karanjalker GR, Ravishankar KV, Shivashankara KS, Dinesh MR, Roy TK, Sudhakar Rao D V. A Study on the Expression of Genes Involved in Carotenoids and Anthocyanins During Ripening in Fruit Peel of Green, Yellow, and Red Colored Mango Cultivars. Appl Biochem Biotechnol. 2018;184(1):140-154. doi:10.1007/s12010-017-2529-x

5. Rodriguez-Amaya DB. Food Carotenoids : Chemistry, Biology and Technology.

6. Ajila CM, Jaganmohan Rao L, Prasada Rao UJS. Characterization of bioactive compounds from raw and ripe Mangifera indica L. peel extracts. Food Chem Toxicol. 2010;48(12):3406-3411. doi:10.1016/J.FCT.2010.09.012

7. Serna-Cock L, García-Gonzales E, Torres-León C. Agro-industrial potential of the mango peel based on its nutritional and functional properties. Food Rev Int. 2016;32(4):364-376. doi:10.1080/87559129.2015.1094815

8. Shirsath SR, Sonawane SH, Gogate PR. Intensification of extraction of natural products using ultrasonic irradiations-A review of current status. Chem 
9. Singh D, Puri M, Wilkens S, Mathur AS, Tuli DK, Barrow CJ. Characterization of a new zeaxanthin producing strain of Chlorella saccharophila isolated from New Zealand marine waters. Bioresour Technol. 2013;143:308-314. doi:10.1016/J.BIORTECH.2013.06.006

10. Dominguez H. Functional Ingredients from Algae for Foods and Nutraceuticals. Elsevier Science; 2013.

11. Marathe SJ, Jadhav SB, Bankar SB, Singhal RS. Enzyme-Assisted Extraction of Bioactives. In: Food Bioactives. Cham: Springer International Publishing; 2017:171-201. doi:10.1007/978-3-319-51639-4_8

12. Serna-Cock L, Torres-León C, Ayala-Aponte A. Evaluación de Polvos Alimentarios obtenidos de Cáscaras de Mango (Mangifera indica) como fuente de Ingredientes Funcionales. Inf tecnológica. 2015;26(2):41-50. doi:10.4067/S071807642015000200006

13. Quintero V, Giraldo G, Lucas J, Vasco J. Caracterización fisicoquímica del mango comun (mangifera indica 1.) durante su proceso de maduración. Biotecnol en el Sect Agropecu y Agroindustrial. 2013;11(1):1-9.

http://www.scielo.org.co/pdf/bsaa/v11n1/v11n1a02.

14. Hong Y-H, Jung EY, Park Y, et al. Enzymatic Improvement in the Polyphenol Extractability and Antioxidant Activity of Green Tea Extracts. Biosci Biotechnol Biochem. 2013;77(1):22-29. doi:10.1271/bbb.120373

15. Sogi DS, Siddiq M, Greiby I, Dolan KD. Total phenolics, antioxidant activity, and functional properties of 'Tommy Atkins' mango peel and kernel as affected by drying methods. Food Chem. 2013;141(3):2649-2655. doi:10.1016/J.FOODCHEM.2013.05.053

16. Hincapié, G., Vásquez, D., Galicia, V., Hincapié C. Propiedades técnicofuncionales de la fibra dietaría de cáscaras de mango variedad hilacha (mangifera indica 1): efecto del secado por convección. Bioctenologíe en el Sect Agropecu y Agroindustrial. 2014;12(1):153-160.

17. Vasco C, Ruales J, Kamal-Eldin A. Total phenolic compounds and antioxidant capacities of major fruits from Ecuador. Food Chem. 2008;111(4):816823. doi:10.1016/J.FOODCHEM.2008.04.054 
18. Dey S, Rathod VK. Ultrasound assisted extraction of $\beta$-carotene from

Spirulina platensis. Ultrason Sonochem. 2013;20(1):271-276.

doi:10.1016/J.ULTSONCH.2012.05.010

19. Lozano J. Fruit Manufacturing. Springer US; 2006. doi:10.1007/978-0-38730616-2

20. Koubala BB, Kansci G, Mbome LI, Crépeau M-J, Thibault J-F, Ralet M-C. Effect of extraction conditions on some physicochemical characteristics of pectins from "Améliorée" and "Mango" mango peels. Food Hydrocoll. 2008;22(7):13451351. doi:10.1016/J.FOODHYD.2007.07.005

Calderón Sara ${ }^{1}$; Vera Edwin ${ }^{1}$

${ }^{1}$ Escuela Politécnica Nacional, Facultad de Ingeniería Química y Agroindustria, Quito, Ecuador 